\title{
PAM4 48-Gbit/s Wireless Communication Using a Resonant Tunneling Diode in the 300-GHz Band
}

\author{
Atsushi Oshiro $^{1 \mathrm{a})}$, Naoki Nishigami ${ }^{1}$, Takumi Yamamoto ${ }^{1}$, Yosuke Nishida ${ }^{2}$, Julian Webber ${ }^{1}$, Masayuki Fujita ${ }^{1 \mathrm{~b})}$, and \\ Tadao Nagatsuma ${ }^{1 \mathrm{c})}$
}

Abstract In modern optical communications, pulse amplitude modulation 4 (PAM4) is employed to achieve higher data rates than that achieved by conventional non-return-to-zero format. Meanwhile, there is an increasing interest to convert wired connections to wireless in data centers using high-speed millimeter-wave and terahertz (THz) links. Here, we introduced the PAM4 modulation for $\mathrm{THz}$ wireless communications using a resonant tunneling diode (RTD) receiver. Compared with a Schottky-barrier diode receiver, the RTD receiver has higher sensitivity, and a stronger nonlinearity at low input power when it is operated with an amplified detection scheme. We achieved 24-Gbaud (48-Gbit/s) transmission in the $300-\mathrm{GHz}$ band with a quasi-real-time digital signal processing (DSP), which is the fastest PAM4 wireless communication without an offline DSP to the best of our knowledge.

key words: terahertz, PAM4, resonant tunneling diode, amplified detection, predistortion.

Classification: $\mathrm{THz}$ devices, circuits and modules

\section{Introduction}

Recently, data traffic has rapidly increased owing to the widespread use of smartphones and the increase in Internet content [1]. In 2017, the IEEE established a 400-Gbit/s Ethernet standard using a pulse amplitude modulation 4 (PAM4) scheme [2]. The PAM4 scheme has four voltage levels and transmits two bits per code [3], which is superior to the conventional non-return-to-zero (NRZ) format with respect to the spectral efficiency, although it requires a larger signal-to-noise ratio (SNR). Compared with other complex modulation schemes such as quadrature phase shift keying (QPSK) and quadrature amplitude modulation (QAM), the PAM4 is particularly suitable for data center interfaces owing to its simplicity, low power consumption, and cost effectiveness [4-8].

Most recently, data centers have faced a practical problem

\footnotetext{
${ }^{1}$ Graduate School of Engineering Science, Osaka University, Toyonaka, Japan

${ }^{2}$ ROHM Co., Ltd., 21 Saiin Mizosaki, Ukyo, Kyoto, 615-8585, Japan.

a) u018625c@ecs.osaka-u.ac.jp

b) fujita@ee.es.osaka-u.ac.jp

c) nagatuma@ ee.es.osaka-u.ac.jp
}

DOI: $10.1587 /$ elex.18.20210494

Received November 22, 2021

Accepted November 29, 2021

Publicized December 07, 2021 related to the wiring complexity and power consumption because of the increase in the scale of facilities [9] [10]. As a promising solution to overcome this issue, wireless interconnection has been proposed [11]. Several studies have been conducted on high-speed wireless links using highfrequency carriers such as millimeter-waves and terahertz (THz) waves [12-15].

$\mathrm{THz}$ waves are electromagnetic waves ranging from 100 $\mathrm{GHz}$ to $10 \mathrm{THz}$, and research on wireless links using 200$\mathrm{GHz} \sim 400-\mathrm{GHz}$ carrier frequencies has been the most active because of the availability of electronics and photonicsbased devices. Photonics-based approaches, where fiberoptic components can be used together with $\mathrm{THz}$ photodiodes in transmitters, have demonstrated $50 \mathrm{Gbit} / \mathrm{s}$ with NRZ modulation and over 100-Gbit/s with higher-order modulation schemes such as QPSK and 16QAM [16-20].

To apply $\mathrm{THz}$ wireless links to inter-rack and inter-base communications in data centers, we studied the use of PAM4 modulation using a photonics-based transmitter and a Schottky-barrier diode (SBD) as a direct detection receiver [21]. We found that the nonlinear response of the system caused the distorted signal and affected the transmission performance. Therefore, we introduced a digital predistortion (DPD) [22] technique to compensate for the signal. However, because of the strong nonlinear response due to the combination of SBD and optical intensity modulator, the data rate is limited to 14-Gbit/s (7-Gbaud) under forward error correction (FEC) communication (FEC limit; bit error-rate $\left.(\mathrm{BER})<2.0 \times 10^{-3}\right)$ [21].

Other research groups have reported THz PAM4 communication using offline DSP [23]. The offline DSP has considerably improved the communication quality, and a communication speed of $60 \mathrm{Gbit} / \mathrm{s}$ (30 Gbaud) has been achieved. Although it is important to show the potential of the technology, it is difficult for other researchers to follow up on the experimental results because various proprietary algorithms are used, which are black boxes. In addition, the real-time nature of the constellation and BER is essential for finding problems in the hardware (the front-end of transmission and reception) in communication experiments. In contrast, experiments and evaluations using a "common" signal processing tool installed in a real-time oscilloscope, etc., al- 
though in quasi real-time, can solve the above problems and is considered to be an important step toward practical use. Resonant tunneling diodes (RTDs) are small $\left(<1 \mathrm{~mm}^{2}\right)$ and have low power consumption $(<20 \mathrm{~mW})$, and have a negative differential conductance (NDC) region, which allows them to oscillate at $\mathrm{THz}$ frequencies and operate as amplifiers [2427]. The nonlinearity of the RTD also enables it to be used as a $\mathrm{THz}$ receiver. Most recently, a coherent detection using the injection-locking phenomenon of RTD has successfully used for wireless communications at $30 \mathrm{Gbit} / \mathrm{s}$ because of its high sensitivity [28] [29].

In this study, we employ the RTD as a receiver instead of the SBD for a simple and cost-effective PAM4 communication system with quasi real-time DSP in the 300-GHz band. By comparing the performance of RTD receiver and SBD one, we show the superiority of the RTD receiver. First, we describe a configuration of the RTD receiver and discuss several detection methods including an amplified detection which is used in our experiments. Then, we explain an experimental setup of the $\mathrm{THz}$ wireless link, which consists of $1.55 \mu \mathrm{m}$ fiber-optic components such as lasers, modulators and photodiodes. Next, we experimentally compare the relative performances of the system using the RTD receiver and the SBD one, and show the superiority of the RTD. Finally, we describe the high-speed wireless communication experiments using the RTD at the data rate of $48 \mathrm{Gbit} / \mathrm{s}$.

\section{Configuration of the RTD receiver}

Fig. 1(a) shows a photograph of the fabricated RTD receiver, which consists of an RTD, a bowtie antenna, a coplanar stripline (CPS), a metal-insulator-metal (MIM) capacitor, and shunt resistor.The thickness of RTD chip is $200 \mu \mathrm{m}$, and the length and width are $550 \mu \mathrm{m}$. The CPS is placed between the MIM capacitor and RTD, and between the RTD and bowtie antenna to match the impedance and adjust the oscillation frequency and phase. The circuit model of the RTD receiver is shown in Fig. 1(b). The oscillation frequency of the RTD used is between $324 \mathrm{GHz}$ and $327 \mathrm{GHz}$. The baseband circuit for the RTD provides a bias voltage and data signals via wire bonding. The circuit is a grounded coplanar waveguide (GCPW) with a $2.4 \mathrm{~mm}$ end-launcher connector attached. A silicon ( $\mathrm{Si}$ ) super- hemispherical lens with a radius of $6 \mathrm{~mm}$ and an offset length of $2.16 \mathrm{~mm}$ is attached to the backside of the chip. The combined directivity of the bowtie antenna and the Si lens is approximately 27 $\mathrm{dBi}$.

In this experiment, the bias voltage applied to the RTD is outside of the oscillation region in the absence of an input signal, but the effective voltage remains inside the region on receiving an input signal with sufficient amplitude.

\section{Operation principle}

The RTD receiver had three detection modes (Fig. 2). The first is direct detection, which is the most basic detection

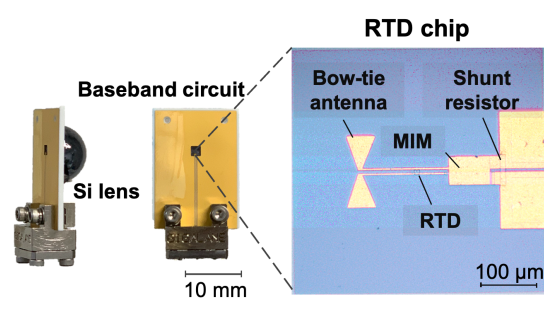

(a)

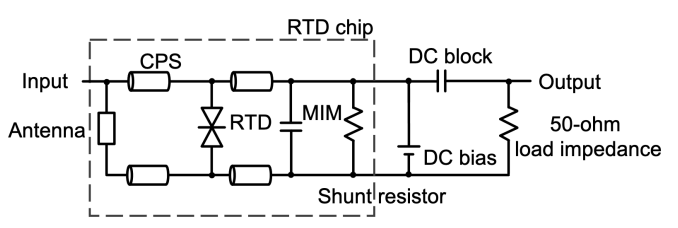

(b)

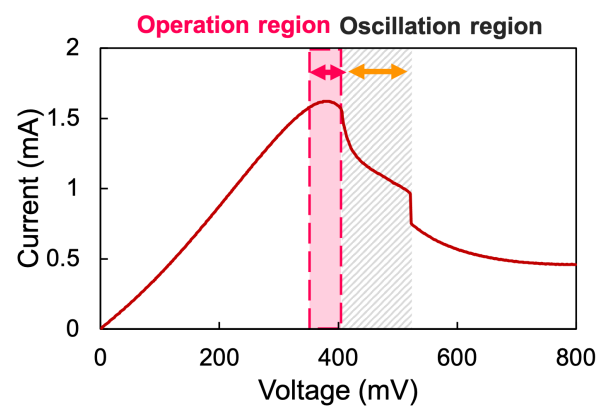

(c)

Fig. 1: RTD receiver. (a) Photo of the fabricated RTD. (b) Circuit model of the receiver. (c) $I-V$ characteristics of the RTD.

method [25] [26]. In the region ( I ), where the outside of the self-oscillation region and the NDC region is far away, only envelope detection using the nonlinearity of the RTD occurs. The second is coherent detection, which operates by applying a bias voltage in the self-oscillation region (region( II )) [28] [29]. Homodyne detection is performed by combining the injection-locking phenomenon and selfoscillating mixing operation.

Amplified detection is the third detection technique after direct detection and coherent detection. When a bias voltage is applied between the two regions (region(III)), amplified detection occurs. Amplified detection is a technique that combines the amplification operation of an RTD, using negative resistance, with the envelope detection. As shown in the previous study [27], by applying a bias voltage outside the negative resistance region and inputting a signal with sufficient amplitude, an effective bias voltage is applied to the negative resistance, and the signal is amplified without oscillation.

The amplified detection can detect signals more than $6 \mathrm{~dB}$ higher in amplitude than coherent detection owing to the difference of the modulation depth. In coherent detection, the oscillation of the RTD is matched with the carrier signal by 


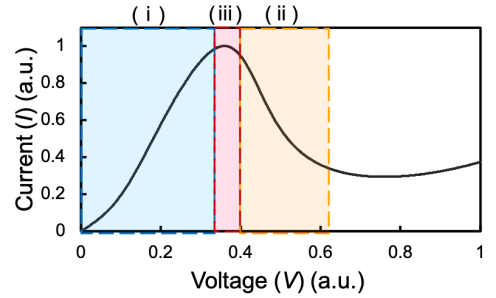

(a)

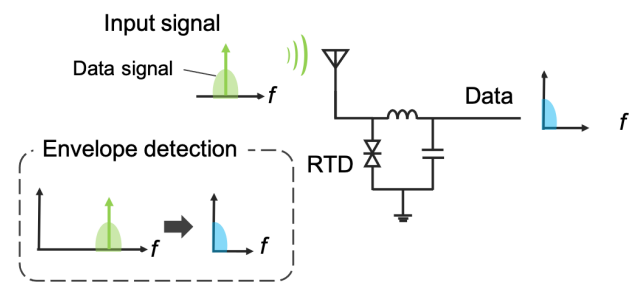

(b)

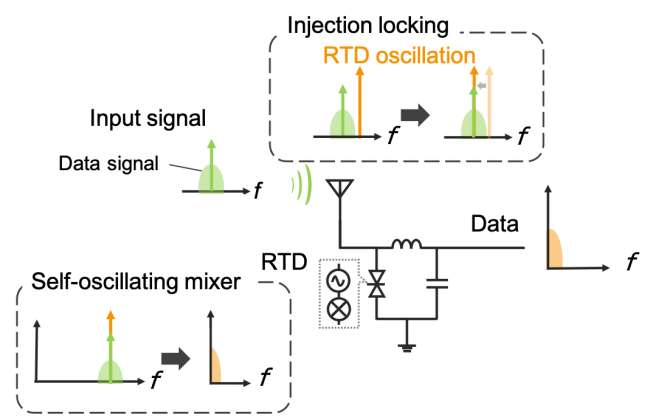

(c)

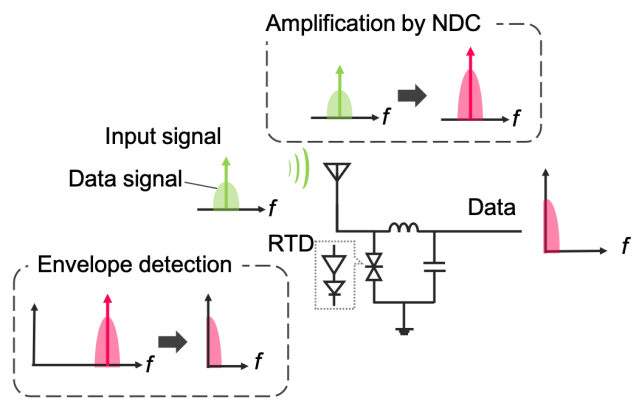

(d)

Fig. 2: Three types of detection. (a) Definition of the operation region in DC $I-V$ characteristics. Operation principles of (b) direct detection (region ( $i$ )), (c) coherent detection (region (ii)), and (d) amplified detection (region (iii)).

injection locking, and only the carrier frequency component is amplified. Therefore, the modulation level inside the RTD is as low as a few tens of percent. In contrast, the amplified detection maintains a high modulation depth because it amplifies all the frequency components of the input signal. This difference provides an advantage of the amplified detection.

Fig. 3 shows a schematic diagram of the input-output characteristics in each detection mode. In the case of direct detection and coherent detection, the slopes become two and one, respectively, and is gradually saturated. In the case of amplified detection, When the input power is small, the slope is approximately two, which indicates direct detection, and the output power increases rapidly as the input power is increased because of amplification by the RTD. When the input power is further increased, the slope decreases below one, and is conjectured to be due to the saturation of the RTD.

The input and output characteristics and their derivative are analyzed by circuit simulation, as shown in Fig. 4 . The equivalent circuit used in the simulation is the same as in Fig. 1(b) and the RTD model is based on previous studies [30]. A steep output power change between $-55-\mathrm{dBm}$ and $50-\mathrm{dBm}$ input power is caused at the beginning of amplified detection, which could make the PAM4's lower eye (between logic level 0 and 1) more open. This result corresponds to that shown in Fig. 3(c) and confirms the measured results. The detection gain was approximately $4 \mathrm{~dB}$. The input power, at which the amplified detection starts, can be controlled by changing the bias voltage. The bias voltage is determined such that the amplification detection starts between logic levels 0 and 1 .

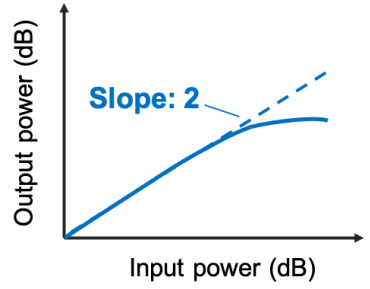

(a)

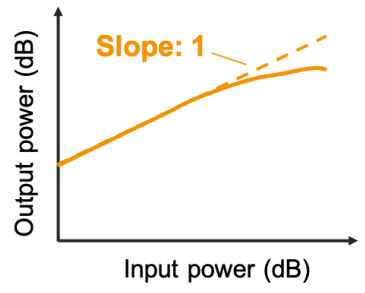

(b)

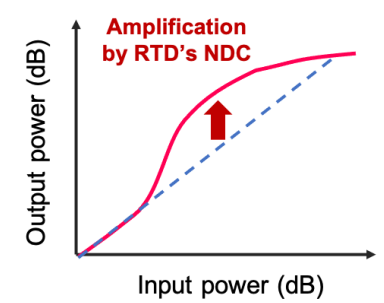

(c)

Fig. 3: Input-output characteristics in each detection mode. (a) Direct detection (region( $\mathrm{i}$ )), (b) coherent detection region(ii), and (c) amplified detection (region(iii)).

\section{Experimental setup}

A block diagram of the PAM4 wireless link system is shown in Fig. 5. Two wavelength-tunable free-running lasers generate optical signals with different wavelengths of $\lambda_{1}$ and $\lambda_{2}$. Then, the optical signals are modulated with the optical intensity modulator (IM), which is driven by the PAM4 signal generated from an arbitrary waveform generator (AWG). After amplifying the optical signals with an erbium-doped 


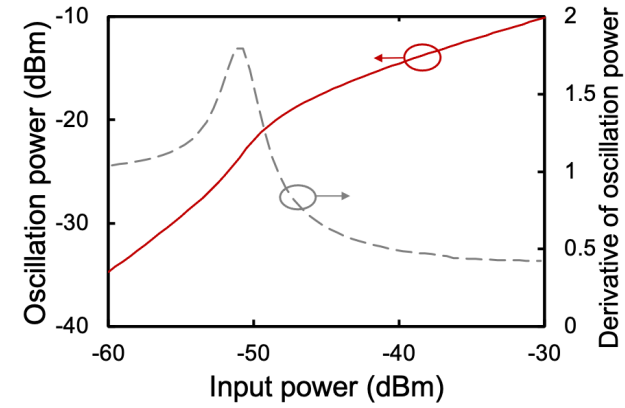

Fig. 4: Simulated input-output characteristics of the RTD. Solid red line is oscillation power and gray dotted line is derivative of oscillation power.

fiber amplifier (EDFA), a uni-travelling-carrier photodiode (UTC-PD) performs optical-to-electrical conversion, and finally, THz waves are radiated from the horn antenna. Transmitted $\mathrm{THz}$ waves are detected by a receicer using a SBD or a RTD and are amplified by a low-noise amplifier (LNA) with an 18-GHz bandwidth; they can be observed on a real time oscilloscope (RTO: Keysight Technologies, DSAZ504A). Quasi real-time waveform compensation is also performed with an equalizer function installed in the RTO. Here, quasi real-time here means that the received data in the processing block are continually processed and BER is continually updated while being accumulated. The transmission distance is set to be $5 \mathrm{~mm}$.

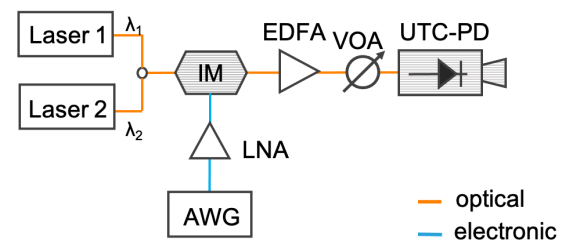

(a)

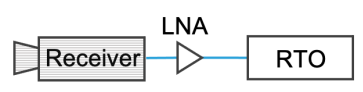

(b)

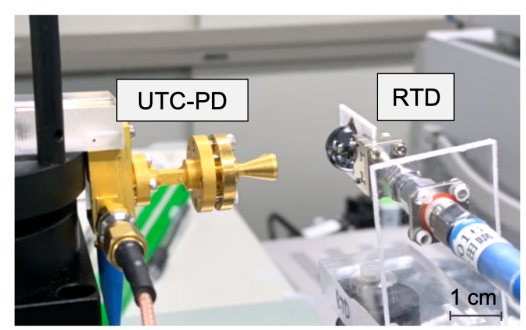

(c)

Fig. 5: System diagram of PAM4 wireless communication in the $300 \mathrm{GHz}$ band. (a) Transmitter side. (b) Receiver side. (c) Photograph of the experimental setup.

We tested and compared a conventional SBD receiver with an RTD receiver in terms of output amplitude and linear- ity, as shown in Fig. 6. This result represents the linearity of the entire system shown in Fig. 5, which includes not only the nonlinearity of the receiver but also the nonlinearity of the optical intensity modulator. As can be seen, the RTD receiver has a higher sensitivity than the SBD receiver, with the received signal amplitude being more than twice as large as that of the SBD receiver. In addition, the RTD receiver is highly responsive at low input power because of the amplification phenomenon described in Section 3, and the difference between each level, when the amplitude is divided into four, becomes more uniform. These features indicate that the RTD receiver is preferable for PAM4, which requires a higher SNR and higher receiver linearity.

\section{Experimental results and discussion}

First, we compared the communication quality of the RTD and SBD as receivers at the same data rate. The data rate was 7 Gbaud (14 Gbit/s). To improve the distortion of the PAM4 received signal and to compensate for the communication quality, we used a technique called pre-distortion [22]. It compensates for the waveforms at the receiver side by making the voltage level spacing of each signal non-uniform (to cancel out the nonlinearity of the system) when PAM4 signals are generated at the transmitter side.

Fig. 7(a) shows the results of the communication experiments with the SBD as an envelope direct detector. The carrier frequency was set to $343 \mathrm{GHz}$, to make use of the largest available radio frequency (RF) bandwidth for both UTC-PD and SBD. As can be seen, the overall noise is high owing to the low sensitivity. The upper eye is particularly affected by the noise. The reason for this is that to compensate for the nonlinearity of the SBD receiver shown in the Fig. 8, the distance between signal levels 0 and 1 is widened by predistortion, which significantly distorts the transmitted waveform (Fig. 8). The BER was $1.68 \times 10^{-3}$, which is below the FEC achievable rate; hence, all the data could be recovered error-free with error correction.

In contrast, the eye pattern of the case with the RTD as receiver is shown in Fig. 7(b). Owing to the high sensitivity, the amplitude becomes larger, and the influence of noise reduces. Comparing the amount of noise at each signal level, the noise at logic level 1 is the largest. This is because the input power at which the amplified detection starts, matches the power at logic level 1 , and is the point at which the output power suddenly increases. The BER is less than $\times 10^{-5}$. The results of the communication experiments show that the RTD receiver is superior to the SBD receiver.

The results of the high-speed communication experiment are shown in Fig. 9. The transmission rate was 24 Gbaud ( $48 \mathrm{Gbit} / \mathrm{s}$ ), and we used predistortion. This is approximately 3.4 times higher data rate than the previous study using the SBD receiver [21]. The predistortion pattern is shown in Fig. 10. The three eyes were opened almost equally, and the BER was measured at $1.98 \times 10^{-3}$.

One of the factors limiting the data rate is the lack of band- 


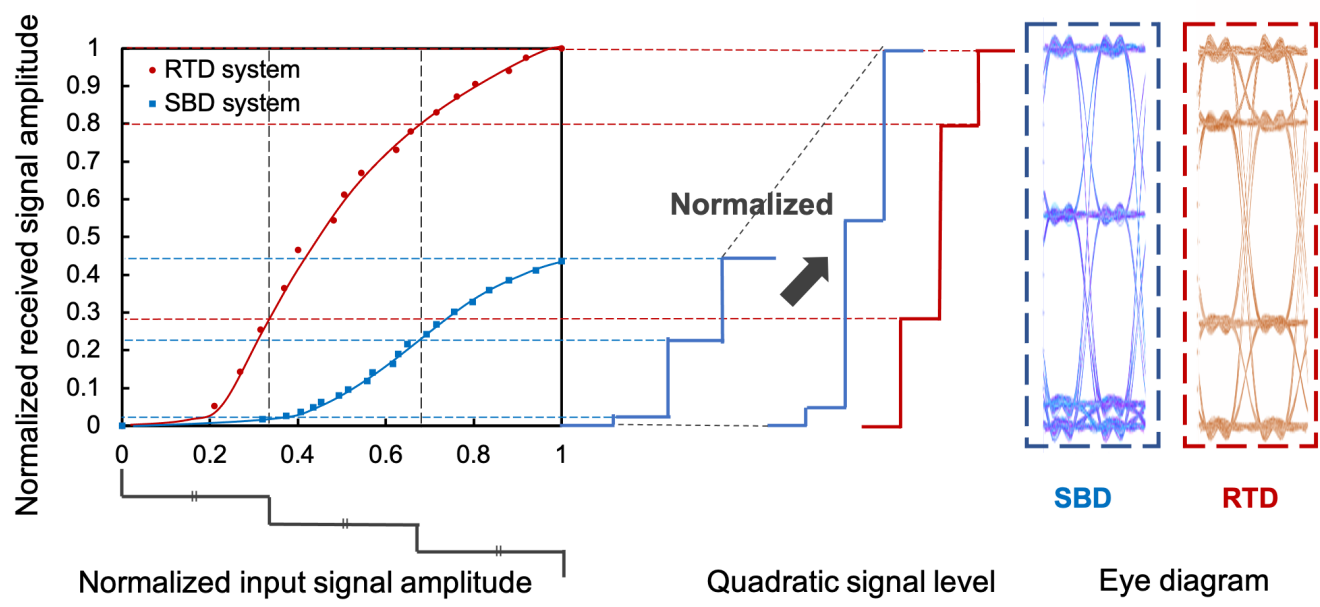

Fig. 6: Relationship between input amplitude and received amplitude of the RTD and SBD schemes. The schematic waveform of the demodulated signal when the four signal levels are equally spaced is shown.

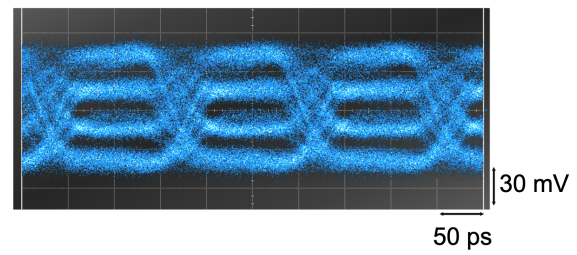

(a)

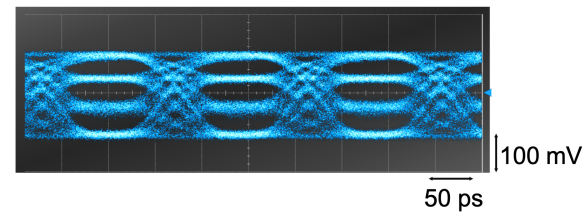

(b)

Fig. 7: Demodulated eye diagrams at 7 Gbaud. (a) Direct detection with SBD. (b) Amplified detection with RTD.

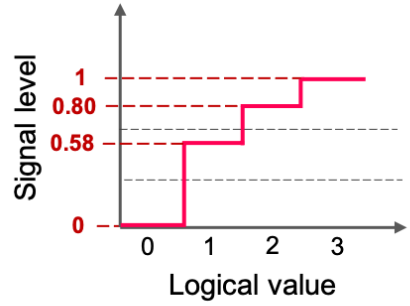

(a)

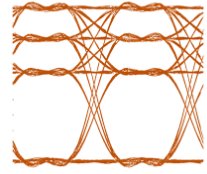

(b)
Fig. 8: Pattern diagram of pre-distortion of SBD. (a) Signal level. (b) Eye diagram.

width in the system. The use of amplifiers with a low noise figure is also important for PAM4 modulation, which lim-

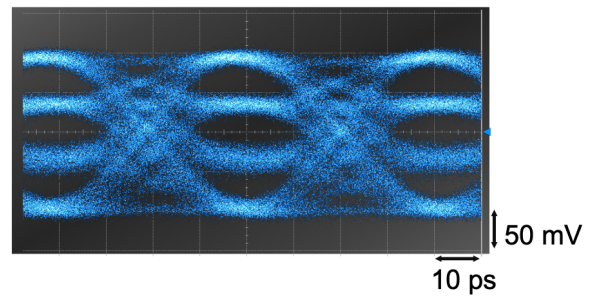

Fig. 9: Demodulated eye diagram of amplified detection using RTD at 24 Gbaud.

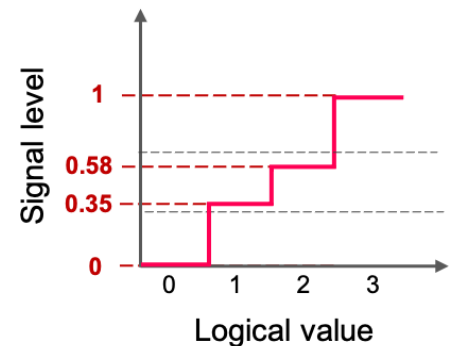

(a)

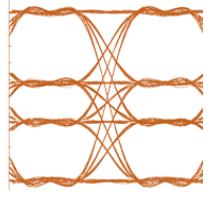

(b)
Fig. 10: Pattern diagram of predistortion of RTD. (a) Signal level. (b) Eye diagram.

its the bandwidth. To further increase the communication speed, it is necessary to increase the sensitivity of the RTD so that amplifiers with a wide bandwidth can be used.

\section{Conclusion}

A PAM4 modulation wireless communication using a $\mathrm{THz}$ RTD receiver was successfully demonstrated. We have achieved $24 \mathrm{Gbaud}(48 \mathrm{Gbit} / \mathrm{s})$ using the RTD receiver with 
quasi real-time DSP, which is the fastest PAM4 wireless link without an offline DSP to the best of our knowledge. Through a comparison of sensitivity, linearity, and communication quality, we showed that the RTD can achieve better results than SBD by efficiently utilizing amplified detection, and that the RTD receiver is suitable for PAM4 modulation wireless communications. As future work, we will perform more detailed analysis on the amplified detection to optimize the THz RTD receiver design including antennas towards over-100-Gbit/s-PAM4 wireless communication.

\section{Acknowledgments}

This work was supported in part by the Core Research for Evolutional Science and Technology (CREST) program of the Japan Science and Technology Agency (JPMJCR1534 and JPMJCR21C4).

\section{References}

[1] Cisco: "Cisco visual networking index (VNI) global mobile data traffic forecast update, 2017-2022" White paper (2019) $1-19$.

[2] C. Cole, et al.: " $400 \mathrm{~Gb} / \mathrm{s} 8 \times 50 \mathrm{G}$ PAM4 WDM $2 \mathrm{~km} \mathrm{SMF}$ PMD baseline specifications," IEEE 802.3 Interim Meeting (2015).

[3] Intel. AN 835.: "PAM4 Signaling Fundamentals," Application Note (2019).

[4] L. Sun, et al.: "Dyadic probabilistic shaping of PAM4 and PAM-8 for cost-effective VCSEL-MMF optical Interconnection," IEEE Photon. J. 11(2)(2019). (DOI: 10.1109/JPHOT.2019.2905011)

[5] A. Healey, et al.: "Beyond 25 Gbps: A study of NRZ \& multilevel modulation in alternative backplane architectures," DesignCon (2013) 1-14.

[6] R. Cideciyan, et al.: "Next generation backplane and copper cable challenges," IEEE Communications Magazine 51(12) (2013) 130-136. (DOI: 10.1109/MCOM.2013.6685768)

[7] C. Morgan, and A. Healey: “A Comparison of 25 Gbps NRZ \& PAM-4 Modulation Used in Reference, Legacy, \& Premium Backplane Channels, International Symposium on Microelectronics (2012) 283-294.

[8] I. Lyubomirsky, and W. A. Ling: "Advanced modulation for datacenter interconnect," 2016 Optical Fiber Communications Conference and Exhibition (2016) 1-3. (DOI: 10.1364/ofc.2016.w4j.3)

[9] A. S. Hamza, et al.: "Wireless communication in data centers: A survey,” IEEE Comcommun Survey mtutorial 18(3) (2016) 1572-1595. (DOI: 10.1109/COMST.2016.2521678)

[10] R. Pries, et al.: "Power consumption analysis of data center architectures," Lecture Notes of the Institute for Computer Sciences, Social-Informatics and Telecommunications Engineering (2015) 114-124. (DOI: 10.1007/978-3-642-333682_10)

[11] Y. Cui, et al.: "Wireless data center networking," IEEE Wireless Commun. 18(6) (2011) 46-53. (DOI: 10.1109/MWC.2011.6108333)

[12] A.Davy, et al.: "Building an end user focused $\mathrm{THz}$ based ultra high bandwidth wireless access network: The TERAPOD approach,” International Congress on Ultra Modern Telecommunications and Control Systems and Workshops (2017) 454-459. (DOI: 10.1109/ICUMT.2017.8255205)

[13] C. Cheng and A. Zajić: "Characterization of propagation phenomena relevant for $300 \mathrm{GHz}$ wireless data center links,"
IEEE Trans. Antennas Propag. 68(2) (2020) 1074-1087. (DOI: 10.1109/TAP.2019.2949135)

[14] C. Terzi, and I. Korpeoglu: "60 GHz wireless data center networks: A survey," Computer Networks 185 (2021). (DOI: 10.1016/j.comnet.2020.107730)

[15] B. Napier: "Terahertz-based ultra-high bandwidth wireless access networks Workshop : From Evolution to Revolution," (2020) $1-4$.

[16] T. Nagatsuma, et al.: "Enabling technologies for real-time 50-Gbit/s wireless transmission at $300 \mathrm{GHz}$," Proceedings of the Second Annual International Conference on Nanoscale Computing and Communication 10 (2015) 1-5. (DOI: $10.1145 / 2800795.2800824)$

[17] S. Jia, et al.: "0.4 THz Photonic-Wireless Link With 106 Gb/s Single Channel Bitrate," J. Lightwave Technol. 36 (2018) 610-616.

[18] T. Kawanishi, "THz and photonic seamless communications," J. Lightwave Technol. 37(7) (2019) 1671-1679. (DOI: 10.1109/JLT.2019.2897042)

[19] C. Castro, et al.: "100 Gbit/s terahertz-wireless realtime transmission using a broadband digital-coherent modem," IEEE 2nd 5G World Forum (2019) 399-402. (DOI: 10.1109/5GWF.2019.8911673)

[20] M. Sung et al.: "Design considerations of photonic $\mathrm{THz}$ communications for $6 \mathrm{G}$ networks," IEEE Wireless Communications (2021) 1-7. (DOI: 10.1109/MWC.001.2100002)

[21] A. Oshiro, et al.: "300-GHz-band wireless communication using pulse amplitude modulation-4," Proceedings of the IEICE General Conference (2020) C-14-4.

[22] Y. Kim, et al.: "A Millimeter-wave CMOS transceiver with digitally pre-distorted PAM-4 modulation for contactless communications," IEEE J. Solid-State Circuits 54(6) (2019), 1600-1612. (DOI: 10.1109/JSSC.2019.2896413)

[23] S. -R. Moon, et al.: “Cost-effective photonics-based THz wireless transmission using PAM-N signals in the $0.3 \mathrm{THz}$ band,” J. Lightwave Technol. 39(2) (2020) 357-362. (DOI: 10.1109/JLT.2020.3032613)

[24] L. L. Chang, et al.: "Resonant tunneling in semiconductor double barriers," Appl.Phys. Lett. 24(12) (1974) 593-595. (DOI: 10.1063/1.1655067)

[25] T. Shiode, et al.: "Giga-bit wireless communication at 300 GHz using resonant tunneling diode detector," Asia-Pacific Microwave Conference Proceedings (2011) 1122-1125.

[26] S. Diebold, et al.: "High-speed error-free wireless data transmission using a terahertz resonant tunnelling diode transmitter and receiver," Electron. Lett. 52(24) (2016) 1999-2001. (DOI: 10.1049/el.2016.2941)

[27] J. Lee and K. Yang,: "RF Power analysis on $5.8 \mathrm{GHz}$ lowpower amplifier using resonant tunneling diodes," IEEE Microw. Wirel. Compon. Lett. 27(1) (2017) 61-63.(DOI: 10.1109/LMWC.2016.2629984)

[28] N. Nishigami, et al.: "Resonant tunneling diode receiver for coherent terahertz wireless communication," Proceedings of 2018 Asia-Pacific Microwave Conference TH4-C1-2 (2018) 726-728.

[29] Y. Nishida, et al.: "Terahertz coherent receiver using a single resonant tunnelling diode," Sci. Rep. 9(1) (2019) 1-3. (DOI: 10.1038/s41598-019-54627-8)

[30] S. Diebold et al.: "Modeling and simulation of terahertz resonant tunneling diode-Based circuits," IEEE Trans. Terahertz Sci. Technol. 6(5) (2016) 716-723. (DOI: 10.1109/TTHZ.2016.2592180) 